\title{
The Role of Lys Side Chains in the Stabilization of $\beta$-Turn Structure
}

\author{
Hiroshi ISHII, Tomoko OKANO,* N. Joe MAEJI,* \\ Yoshio INOUE,* and Riichirô CHÛJÔ* \\ Department of Industrial Chemistry, Tokyo National College of Technology, \\ 1220 Kunugida, Hachioji-shi, Tokyo 193, Japan \\ * Department of Polymer Chemistry, Tokyo Institute of Technology, \\ 12-1 Ookayama, Meguro-ku, Tokyo 152, Japan
}

(Received June 10, 1987)

\begin{abstract}
Peptides containing Lys and Orn were synthesized to study the role of side chains in the formation of the $\beta$-turn structure. In contrast with peptides having a -Lys-Asp- sequence, peptides containing an -Orn-Asp- sequence do not adopt a $\beta$-turn structure. Analysis of the side chain rotamers shows that in Boc-Gly-Lys-Asp-Gly-OMe the Lys side chain can interact with the side chain of the neighboring Asp residue and this interaction contributes to the stabilization of the $\beta$-turn structure. The Orn side chain, instead of side chain-side chain interactions, interacts with the peptide backbone.
\end{abstract}

KEY WORDS $\beta$-Turn Structure / Side Chain Conformation / Hydrogen Bond / Intramolecular Interaction /

The reverse turn of peptide chains is an important structural feature of proteins, ${ }^{1-3}$ since many biological events, such as protein folding, ${ }^{4,5}$ occur at a so-called $\beta$-turn region. The $\beta$-turn structure, consisting of four amino acid residues, $i, i+1, i+2$, and $i+3$, appears in short-chain peptides as well as in proteins. ${ }^{6}$ Many efforts have been made to elucidate the origins of the formation and stabilization of $\beta$ turn structures. ${ }^{7-9}$ Besides the intramolecular hydrogen bond between backbone amide groups, additional intramolecular interactions stabilizing the $\beta$-turn structure have been proposed..$^{10,11}$

In previous papers, ${ }^{12,13}$ we showed by NMR study that peptides having -Lys-Asp- sequence favor a $\beta$-turn structure, where Lys and Asp residues are at $(i+1)$ - and $(i+2)$-th positions, respectively. It was also evident that the intramolecular interactions between the Lys and Asp side chains contribute to stabilize the $\beta$-turn structure. Since Lys has a long aliphatic side chain, it is expected that the Lys side chain has some particular conformational feature. In peptides forming a $\beta$-turn structure, however, the conformation of the Lys side chain has not been clarified exactly. For polyL-lysine the rotamer population about the side chain $\mathrm{C}-\mathrm{C}$ bond of the Lys side chain has been calculated. ${ }^{14}$

In this paper, we show a conformational preference of the Lys side chain in peptides. To elucidate the possible role of the Lys side chain in the formation and stabilization of the $\beta$-turn structure, peptides containing Orn are studied for comparison. The Orn residue is found in biologically active peptides, such as gramicidin $\mathrm{S}$, and possesses shorter aliphatic side chain than Lys. Terminal protected norleucine (Nle) and norvaline (Nva) are also studied. These amino acids are related to Lys and Orn, respectively, but lack the side chain terminal amino group. The structure of Lys, Orn, Nle, and Nva residues are shown in Figure 1. The 

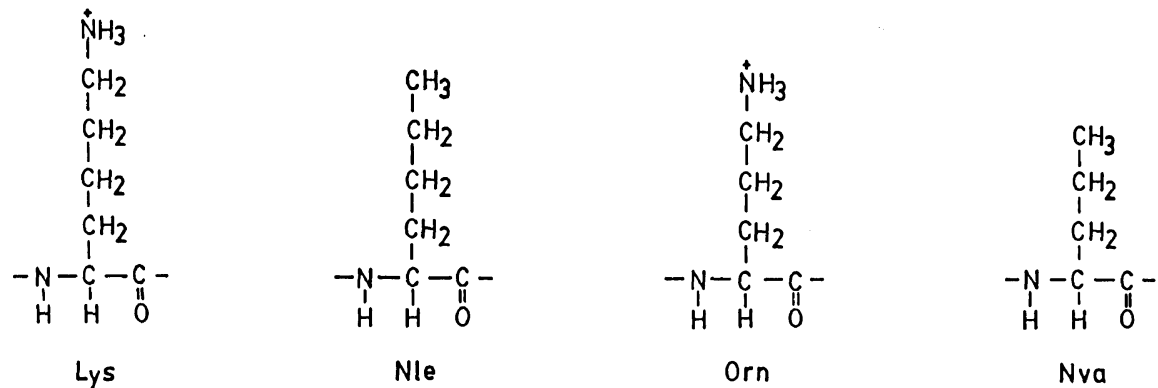

Figure 1. Structures of Lys, Orn, Nle, and Nva residues.

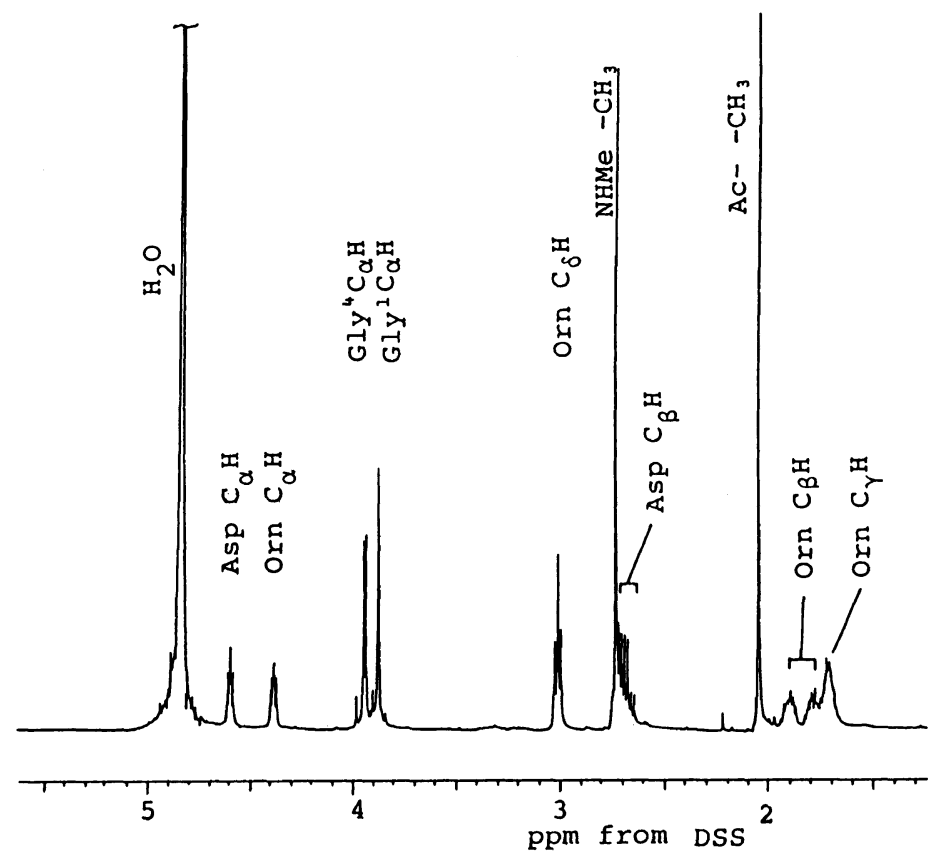

Figure 2. The $500 \mathrm{MHz}{ }^{1} \mathrm{H}$ NMR spectrum of Ac-Gly-Orn-Asp-Gly-NHMe in ${ }^{2} \mathrm{H}_{2} \mathrm{O}$ solution.

peptides investigated are as follws: Ac-LysAsp-NHMe, Boc-Gly-Lys-Asp-Gly-OMe, Ac-Asp-Lys-NHMe, Boc-Gly-Asp-LysGly-OMe, Ac-Orn-Asp-NHMe, Ac-AspOrn-NHMe, Ac-Gly-Orn-Asp-Gly-NHMe, Ac-Lys-NHMe, Ac-Orn-NHMe, Ac-Nle$\mathrm{NHMe}$, and Ac-Nva-NHMe. The former two peptides has been found to take a $\beta$-turn structure. $^{12,13}$

\section{EXPERIMENTAL}

\section{Synthesis of Peptides}

Protected amino acids and peptides were synthesized by the liquid phase method. ${ }^{15}$ All peptides were purified by repetitive recrystallization and were identified by ${ }^{1} \mathrm{H}$ NMR and thin-layer chromatography on silica gel. Amino acids were purchased from Kokusan Kagaku (Tokyo). In Figure 2 is shown the $500 \mathrm{MHz}{ }^{1} \mathrm{H}$ NMR spectrum of Ac-Gly-OrnAsp-Gly-NHMe. 


\section{NMR Measurement}

${ }^{1} \mathrm{H}$ NMR spectra were recorded on a JEOL GX-500 spectrometer operating at $500 \mathrm{MHz}$. Hydrogen oxide and deuterium oxide from Merck Sharp \& Dohme Canada Ltd. were used as solvents. The $\mathrm{pH}$ values of NMR samples were measured by a $\mathrm{pH}$ meter and reported without any correction. Sodium-2,2-dimethyl2-silapentane-5-sulfonate (DSS) and sodium 2,2,3,3-tetradeutero-3-trimethylsilyl propionate (TSP) were used as internal references. Tetramethylsilane (TMS) was also used as an external reference. Spectral assignments were achieved by selective homo-spin decoupling. The prochiral methylenic protons of the Lys and Orn side chains were assigned based on those in the literature ${ }^{12,13,16}$ and the assignments were supported by good agreement of the simulated spectra with the observed spectra.

\section{NMR Spectral Simulation}

The NMR resonances of the Lys and Orn side chains were analyzed by the simulation and iteration program, LAOCON3, using a Hitachi HITAC M-280H computer. For the spin-simulation, the long range couplings, i.e., ${ }^{4} J$ and ${ }^{5} J$ were neglected.

\section{RESULTS AND DISCUSSION}

\section{Formation of the $\beta$-Turn Structure}

For dipeptides containing Lys, it has been found that Ac-Lys-Asp-NHMe adopts a $\beta$ turn-like structure, while Ac-Asp-Lys-NHMe dose not. ${ }^{12}$ Boc-Gly-Lys-Asp-Gly-OMe also tends to take a $\beta$-turn structure in solution. ${ }^{13}$ It is evident that peptides which possess a -LysAsp-sequence favor a $\beta$-turn structure.

For di- and tetrapeptides containing Orn, concentration dependence of the chemical shift $(0.050-0.017 \mathrm{M})$ was not significant for all amide protons, indicating the absence of intermolecular interaction. In Table I are listed the values of the temperature dependence of the amide proton chemical shift $(\mathrm{d} \delta / \mathrm{d} T)$. All amide protons in Ac-Orn-Asp-NHMe and Ac-Asp-Orn-NHMe showed much larger $\mathrm{d} \delta /$ $\mathrm{d} T$ values than those reported for intramolecularly hydrogen bonded amide protons. ${ }^{17,18}$ This indicates that both Ac-Orn-Asp-NHMe and Ac-Asp-Orn-NHMe take no stable intramolecularly hydrogen bonded conformation in solution. The chemical shifts of the amide protons in Ac-Gly-Orn-Asp-GlyNHMe show greater temperature dependence than those in Boc-Gly-Asp-Lys-Gly-OMe $(\mathrm{d} \delta / \mathrm{d} T$ values in an aqueous solution are $-4.49 \times 10^{-3}, \quad-4.75 \times 10^{-3}, \quad-5.00 \times 10^{-3}$, and $-4.33 \times 10^{-3} \mathrm{ppm} \mathrm{deg}^{-1}$ for $\mathrm{Gly}^{1}$, Asp, Lys, and $\mathrm{Gly}^{4}$ amide protons, respectively), which could not take the $\beta$-turn structure. ${ }^{13}$ This indicates that Ac-Gly-Orn-Asp-Gly$\mathrm{NHMe}$ hardly takes an intramolecular hydrogen bond responsible for the $\beta$-turn structure.

For the peptides investigated, it became evident that peptides containing a -Lys-Aspsequence have a tendency to adopt a $\beta$-turn structure, while the peptides containing an

Table I. Temperature dependence $(293-323 \mathrm{~K})$ of the amide proton chemical shifts $(\mathrm{d} \delta / \mathrm{d} T)$ for Orn-containing peptides measured in $\mathrm{H}_{2} \mathrm{O}_{-}{ }^{2} \mathrm{H}_{2} \mathrm{O}$ system

\begin{tabular}{lccccc}
\hline & \multicolumn{5}{c}{$\mathrm{d} \delta / \mathrm{d} T\left(\times 10^{-3} \mathrm{ppm} \mathrm{deg}^{-1}\right)$} \\
\cline { 2 - 5 } Compound & Asp NH & Orn NH & Gly NH $^{1}$ & Gly $^{4}$ NH & NHMe \\
\hline Ac-Orn-Asp-NHMe & -9.87 & -8.99 & & -7.55 \\
Ac-Asp-Orn-NHMe & -8.92 & -10.26 & -5.81 & -7.09 \\
Ac-Gly-Orn- & -6.14 & -6.81 & -5.84 & -4.52 \\
Asp-Gly-NHMe & & & & \\
\hline
\end{tabular}


(A)
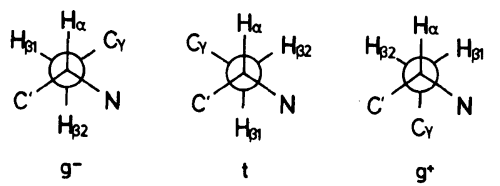

(B)
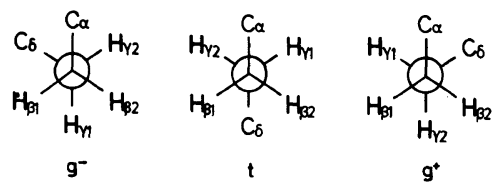

(D)
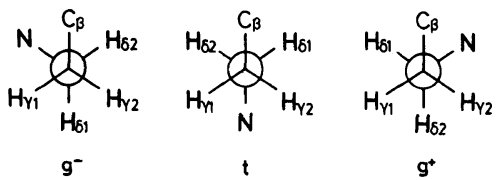

Figure 3. Three rotamers about $\alpha-\beta$ (A) and $\beta-\gamma(\mathrm{B})$ bonds for Lys and Orn side chains. Three rotamers about $\delta-\varepsilon(\mathrm{C})$ and $\gamma-\delta(\mathrm{D})$ bonds for Lys and Orn side chains, respectively.

-Orn-Asp- sequence do not. Since Orn residue lacks only one methylene unit in the side chain compared with Lys residue, the difference in $\beta$-turn forming potential is very interesting. It is probable that the Lys side chain play an important role in the formation and stabilization of the $\beta$-turn structure.

\section{Conformation about the $\alpha-\beta$ Bond for Lys and Orn}

In Figure 3 are shown the three stable rotamers $\left(g^{-}, t\right.$, and $\left.g^{+}\right)$about the $\alpha-\beta, \beta-\gamma, \gamma^{-}$ $\delta$, and $\delta-\varepsilon$ bonds for Lys and/or Orn side chains. Relative populations of the rotamers $\left(P_{g^{-}}, P_{t}\right.$, and $\left.P_{g^{+}}\right)$about the $\alpha-\beta$ bond were calculated from the observed vicinal coupling constants ${ }^{3} J(\mathrm{HCCH})$ using Feeney's approximation. ${ }^{19}$ The vicinal coupling constants and rotamer populations of the peptides containing Lys and Orn at $\mathrm{pH} 3,7$, and 12 are listed in Tables II and III, respectively. The observed titration shift along with previous results ${ }^{12,13}$ indicate that the Asp, Lys, and Orn residues are almost protonated and deprotonated at pH 3 and 12, respectively. At pH 7, the Asp residue is deprotonated, while the Lys and Orn residues are in the protonated form.

As shown in Tables II and III, the populations of the rotamers about the $\alpha-\beta$ bond of
Lys and Orn depend on the ionization state not only of the Lys and Orn side chains but also of the Asp side chain. While the $g^{+}$ rotamer is least populated in most of all

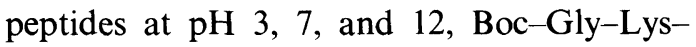
Asp-Gly-OMe showed $P_{g^{+}}$value greater than $P_{t}$ at $\mathrm{pH}$ 7. This consistent with our previous conclusion $^{12,13}$ that there is an intramolecular interaction between the carboxylate and protonated amino groups in the -Lys-Aspsequence.

To elucidate the conformational difference between Lys and Orn, a rotamer analysis of the side chain was done for the $N$-acetyl- $N^{\prime}$ methylamide derivatives of Lys, Orn, Nle, and Nva. For Ac-Lys-NHMe, the deprotonation of the $\varepsilon$-amino group slightly affects the population. The conformation about the $\alpha-\beta$ bond in Ac-Lys-NHMe is similar to that in AcNle-NHMe, indicating that the $\varepsilon$-amino group itself does not participate in intramolecular interaction with peptide backbone (intraresidue interaction). On the other hand, there is a significant change in the rotamer populations in Ac-Orn-NHMe on the deprotonation of the $\delta$-amino group. This suggests the presence of an intramolecular interaction between the $\delta$-amino group and peptide backbone.

The rotamer populations about the $\alpha-\beta$ 
Table II. Proton-proton coupling constants $(\mathrm{Hz})$ and relative rotamer populations about Lys or Nle $\alpha-\beta$ bond for peptides containing Lys and Ac-Nle-NHMe

\begin{tabular}{lrrrrrr}
\hline Compound & $\mathrm{pH}$ & $J_{\alpha \beta_{1}}$ & $J_{\alpha \beta_{2}}$ & $P_{g-}$ & $P_{t}$ & $P_{g+}$ \\
\hline Ac-Lys-Asp-NHMe & 3 & 5.80 & 8.24 & 0.56 & 0.35 & 0.09 \\
& 7 & 6.11 & 7.94 & 0.52 & 0.35 & 0.09 \\
Ac-Asp-Lys-NHMe & 12 & 5.65 & 8.09 & 0.55 & 0.32 & 0.13 \\
& 3 & 5.37 & 9.34 & 0.68 & 0.32 & 0.00 \\
Boc-Gly-Lys- & 7 & 4.58 & 9.77 & 0.75 & 0.25 & 0.00 \\
Asp-Gly-OMe & 12 & 5.04 & 9.31 & 0.69 & 0.29 & 0.02 \\
Boc-Gly-Asp- & 3 & 6.25 & 8.09 & 0.53 & 0.40 & 0.07 \\
Lys-Gly-OMe & 7 & 5.06 & 7.60 & 0.50 & 0.23 & 0.27 \\
Ac-Lys-NHMe & 12 & 5.39 & 8.65 & 0.61 & 0.31 & 0.08 \\
Ac-Nle-NHMe & 3 & $-*$ & $-*$ & & & 0.73 \\
& 7 & 4.43 & 9.62 & 0.73 & 0.22 & 0.05 \\
& 7 & 5.59 & 9.54 & 0.64 & 0.27 & 0.09 \\
\end{tabular}

* Not observed due to overlapping peaks.

Table III. Proton-proton coupling constants $(\mathrm{Hz})$ and relative rotamer populations about Orn or Nva $\alpha-\beta$ bond for peptides containing Orn and Ac-Nva-NHMe

\begin{tabular}{lrlllll}
\hline Compound & $\mathrm{pH}$ & $J_{\alpha \beta_{1}}$ & $J_{\alpha \beta_{2}}$ & $P_{g-}$ & $P_{t}$ & $P_{g+}$ \\
\hline Ac-Orn-Asp-NHMe & 3 & 5.49 & 7.33 & 0.51 & 0.29 & 0.20 \\
& 7 & 5.71 & 7.71 & 0.50 & 0.32 & 0.18 \\
Ac-Asp-Orn-NHMe & 12 & 5.49 & 8.54 & 0.60 & 0.32 & 0.08 \\
& 3 & 5.19 & 8.85 & 0.64 & 0.29 & 0.07 \\
Ac-Gly-Orn- & 7 & 4.58 & 8.85 & 0.65 & 0.21 & 0.14 \\
Asp-Gly-NHMe & 12 & 5.19 & 9.47 & 0.70 & 0.30 & 0.00 \\
Ac-Orn-NHMe & 3 & 5.50 & 8.55 & 0.60 & 0.32 & 0.08 \\
Ac-Nva-NHMe & 7 & 5.65 & 8.40 & 0.58 & 0.34 & 0.08 \\
& 12 & 5.34 & 8.70 & 0.62 & 0.31 & 0.07 \\
\end{tabular}

bond of the Asp side chain changed little on deprotonation of the Orn $\delta$-amino group in the peptides containing Orn.

\section{Conformation of the Side Chain Terminals for Lys and Orn}

The populations of the rotamers about the $\delta-\varepsilon$ and the $\gamma-\delta$ bonds for Lys and Orn side chains, respectively, were calculated using the following equation. ${ }^{14}$

$$
\begin{aligned}
P_{t}= & 4\left(J_{\delta_{\varepsilon}}-J_{g}\right) /\left(J_{t}-J_{g}\right)-1 \\
& \text { for Lys } \delta-\varepsilon \text { bond } \\
P_{t}= & 4\left(J_{\gamma \delta}-J_{g}\right) /\left(J_{t}-J_{g}\right)-1 \\
& \text { for Orn } \gamma-\delta \text { bond }
\end{aligned}
$$

Since two $\varepsilon$ and $\delta$ protons of Lys and Orn, respectively, are equivalent to each other, only the $P_{t}$ value can be estimated. The mean coupling constants and rotamer populations for the peptides containing Lys and Orn are tabulated in Tables IV and V, respectively. 
Table IV. Proton-proton coupling constants $(\mathrm{Hz})$ and relative rotamer populations about Lys $\delta-\varepsilon$ bond for peptides containing Lys

\begin{tabular}{lrccc}
\hline Compound & $\mathrm{pH}$ & $J_{\delta \varepsilon}$ & $P_{t}$ & $P_{g-}+P_{\mathrm{g}+}$ \\
\hline Ac-Lys-Asp-NHMe & 3 & 7.62 & 0.91 & 0.09 \\
& 7 & 7.48 & 0.85 & 0.15 \\
& 12 & 7.18 & 0.72 & 0.28 \\
Ac-Asp-Lys-NHMe & 3 & 7.33 & 0.78 & 0.22 \\
& 7 & 7.10 & 0.68 & 0.32 \\
Boc-Gly-Lys- & 12 & 7.94 & 1.00 & 0.00 \\
Asp-Gly-OMe & 3 & 8.40 & 1.00 & 0.00 \\
& 7 & 7.48 & 0.85 & 0.15 \\
Boc-Gly-Asp- & 12 & 7.33 & 0.78 & 0.22 \\
Lys-Gly-OMe & 3 & 7.48 & 0.85 & 0.15 \\
& 7 & 7.33 & 0.78 & 0.22 \\
Ac-Lys-NHMe & 12 & 6.41 & 0.38 & 0.62 \\
& 7 & 7.63 & 0.91 & 0.09 \\
& 12 & 6.87 & 0.58 & 0.42 \\
\hline
\end{tabular}

Table V. Proton-proton coupling constants $(\mathrm{Hz})$ and relative rotamer populations about Orn $\gamma-\delta$ bond for peptides containing Orn

\begin{tabular}{lrrcc}
\hline Compound & $\mathrm{pH}$ & $J_{\gamma \delta}$ & $P_{t}$ & $P_{g-}+P_{g+}$ \\
\hline Ac-Orn-Asp-NHMe & 3 & 7.02 & 0.65 & 0.35 \\
& 7 & 7.33 & 0.78 & 0.22 \\
& 12 & $-^{*}$ & & \\
Ac-Asp-Orn-NHMe & 3 & 7.33 & 0.78 & 0.22 \\
& 7 & 7.63 & 0.91 & 0.09 \\
Ac-Gly-Orn- & 12 & $-^{*}$ & & \\
Asp-Gly-NHMe & 3 & 7.48 & 0.85 & 0.15 \\
& 7 & 7.48 & 0.85 & 0.15 \\
Ac-Orn-NHMe & 72 & $-^{*}$ & & \\
& 12 & - - $^{*}$ & & \\
\hline
\end{tabular}

* Not observed due to overlapping peaks.
In contrast to the conformation about the $\alpha-\beta$ bond, the terminal conformation of the Lys side chain depends on the ionization state of $\varepsilon$-amino group for all peptides containing Lys but Boc-Gly-Lys-Asp-Gly-OMe. In Boc-Gly-Lys-Asp-Gly-OMe, the terminal conformation of the Lys side chain is greatly affected by deprotonation of the neighboring Asp side chain. On the deprotonation of the Asp side chain, the conformational change about the Lys $\delta-\varepsilon$ bond accompanies that of the Lys $\alpha-\beta$ bond in Boc-Gly-Lys-Asp-GlyOMe. From these results, it can be said that the conformation of the side chain terminal is influenced not by the intramolecular interaction between the Lys side chain and peptide backbone but by the intramolecular interaction between the Lys protonated amino group and the Asp carboxylate group in Boc-GlyLys-Asp-Gly-OMe.

In contrast to Boc-Gly-Lys-Asp-GlyOMe, little change was observed for the Lys $\delta$ $\varepsilon$ bond on the deprotonation of the Asp side chain in Boc-Gly-Asp-Lys-Gly-OMe. It seems likely that there is no intramolecular interaction between Lys and Asp side chains.

Since the coupling constants about $\gamma-\delta$ bond could not be observed for Orn at $\mathrm{pH} 12$, the conformational change of the Orn side chain on the deprotonation of the $\delta$-amino group is not known. In Ac-Gly-Orn-Asp-GlyNHMe, no change was observed in the rotamer population about Orn $\gamma-\delta$ bond on deprotonation of the Asp side chain, indicat-

Table VI. Proton-proton coupling constants $(\mathrm{Hz})$ and relative rotamer populations about Lys $\beta-\gamma$ bond for peptides containing Lys

\begin{tabular}{lrrrrrrrr}
\hline Compound & $\mathrm{pH}$ & $J_{\beta_{1} \gamma_{1}}$ & $J_{\beta_{1} \gamma_{2}}$ & $J_{\beta_{2} \gamma_{1}}$ & $J_{\beta_{2} \gamma_{2}}$ & $P_{g-}$ & $P_{t}$ & $P_{g+}$ \\
\hline Ac-Lys-Asp-NHMe & 3 & $-^{*}$ & $-^{*}$ & $-^{*}$ & $-^{*}$ & & \\
& 7 & 7.50 & 7.50 & 7.00 & 7.00 & 0.16 & 0.74 & 0.10 \\
Ac-Asp-Lys-NHMe & 12 & 6.80 & 6.90 & 8.00 & 6.70 & 0.10 & 0.68 & 0.22 \\
& 3 & 6.00 & 8.00 & 4.50 & 10.00 & 0.18 & 0.69 & 0.13 \\
& 7 & $--^{*}$ & $--^{*}$ & -0.00 & 9.60 & 0.20 & 0.70 & 0.10 \\
\hline
\end{tabular}

* Not observed due to overlapping peaks. 
ing no intramolecular interaction between side chains in Ac-Gly-Orn-Asp-Gly-NHMe.

\section{Conformation about the $\beta-\gamma$ Bond for Lys Side Chain}

The rotamer populations about the $\beta-\gamma$ bond were calculated from the coupling constant ${ }^{3} J(C \beta \mathrm{HC} \gamma \mathrm{H}) .{ }^{14}$ Because of severe peak overlapping, spin simulation could be done only for Ac-Lys-Asp-NHMe and Ac-AspLys-NHMe. In Table VI are listed four vicinal coupling constants about the $\beta-\gamma$ bond and the rotamer population values. For the Lys $\beta-\gamma$ bond, the $t$-rotamer is the most populated, consistent with the results reported for poly-Llysine. ${ }^{14}$ There is no significant rotamer change about the $\beta-\gamma$ bond on $\mathrm{pH}$ change, probably due to the stability of the $t$-rotamer about the $\beta-\gamma$ bond.

\section{CONCLUSIONS}

From analysis of the rotamer populations, several conformational features of the Lys and Orn side chains can be drawn. First, for the Lys $\alpha-\beta$ bond, the $g^{+}$rotamer is the least populated among the peptides investigated except for Boc-Gly-Lys-Asp-Gly-OMe. Second, the conformation about the Lys $\alpha+\beta$ bond in Ac-Lys-NHMe is slightly influenced by the deprotonation of the side chain, while the conformation about the Orn $\alpha-\beta$ bond depends on the ionization state of the side chain in Ac-Orn-NHMe. There is an appreciable difference in the conformational preference in the side chain between the Lys and Orn residues, i.e., the chain length in the side chain affects the degree of the intramolecular interaction. Third, in Boc-Gly-Lys-Asp-Gly$\mathrm{OMe}$, the Lys side chain conformation depends on the ionization state of the neighboring Asp side chain. There is an intramolecular interaction between the Lys protonated amino and Asp carboxylate groups in Boc-Gly-LysAsp-Gly-OMe.

It has been proposed that the peptides which possess a -Lys-Asp- sequence adopt a $\beta$-turn structure, whereas peptides containing an -Orn-Asp- sequence do not. The difference in the $\beta$-turn forming tendency between BocGly-Lys-Asp-Gly-OMe and Ac-Gly-OrnAsp-Gly-NHMe is due to the occurrence of the intramolecular interaction involving the side chains. It seems likely that the Lys side chain plays an important role in adoption of the $\beta$-turn structure in the peptides containing the -Lys-Asp- sequence.

Acknowledgements. We are very grateful to Dr. I. Ando, Tokyo Institute of Technology, for use of computer program LAOCON3. This work was partly supported by a Grant-in-Aid for Scientific Reserch (No. 62430019) from the Ministry of Education, Science, and Culture of Japan.

\section{REFERENCES}

1. C. Venkatachalam, Biopolymers, 6, 1425 (1968).

2. P. Y. Chou and G. D. Fasman, Biochemistry, 13, 222 (1974).

3. P. Y. Chou and G. D. Fasman, J. Mol. Biol., 115, 135 (1977).

4. I. D. Kuntz, J. Am. Chem. Soc., 94, 4009 (1972).

5. P. Y. Lewis, F. A. Momany, and H. A. Scheraga, Proc. Natl. Acad. Sci. U.S.A., 68, 2293 (1971).

6. J. A. Smith and L. A. Pease, CRC Crit. Rev. Biochem., 315 (1980).

7. A. C. Bach and L. M. Gierasch, Biopolymers, 25, S175 (1986).

8. N. Manesis, M. Hassan, R. Glaser, and M. Goodman, Biopolymers, 25, 597 (1986).

9. A. F. Spatola, M. K. Anwer, A. L. Rockwell, and L. M. Gierasch, J. Am. Chem. Soc., 108, 825 (1986).

10. R. Mayer and G. G. Lancelot, J. Am. Chem. Soc., 103, 4738 (1981).

11. M. Marraud and A. Aubry, Int. J. Peptide Protein Res., 23, 123 (1984).

12. H. Ishii, Y. Inoue, and R. Chûjô, Polym. J., 16, 9 (1984).

13. H. Ishii, Y. Fukunishi, Y. Inoue, and R. Chûjô, Biopolymers, 24, 2045 (1985).

14. B. Perly, Y. Chevalier, and C. Chachaty, Macromolecules, 14, 969 (1981).

15. J. C. Howard, A. Ali, H. A. Scheraga, and F. A. Momany, Macromolecules, 8, 607 (1975).

16. A. Bundi and K. Wuthrich, Biopolymers, 18, 285 (1979). 


\section{H. IsHII et al.}

17. M. Iqbal and P. Balaram, Biopolymers, 21, 1427 (1982).

18. R. Deslauries and I. C. P. Smith, "Biological Magnetic Resonances," Vol. 2, L. J. Berliner and J.
Reubem, Ed., Plenum Press, New York, N.Y., 1980, p 243.

19. J. Feeney, J. Magn. Reson., 21, 473 (1976). 